Canadian

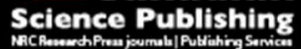

Canadian Journal of Forest Research Revue canadienne de recherche forestière

\title{
Spatially consistent imputations of forest data under a semivariogram model
}

\begin{tabular}{|r|l|}
\hline Journal: & Canadian Journal of Forest Research \\
\hline Manuscript ID & cjfr-2016-0068.R2 \\
\hline Danuscript Type: & Article \\
\hline Complete List of Authors: & $\begin{array}{l}\text { Strimbu, Victor; Norges miljo- og biovitenskapelige universitet, } \\
\text { Department of Ecology and Natural Resource Management; } \\
\text { Ene, Liviu; Norwegian University of Life Sciences, Department of Ecology } \\
\text { and Natural Resource Management } \\
\text { Næsset, Erik; Norwegian University of Life Sciences, Department of } \\
\text { Ecology and Natural Resource Management }\end{array}$ \\
\hline Keyword: & $\begin{array}{l}\text { spatial imputations, autocorrelation, semivariogram, simulation, } \\
\text { optimization }\end{array}$ \\
\hline &
\end{tabular}


4 P.O. Box 5003, N01432 Ås, Norway

5 victor.strimbu@nmbu.no

$6 \quad$ Liviu Teodor Ene

7 Department of Ecology and Natural Resource Management, Norwegian University of Life Sciences,

$8 \quad$ P.O. Box 5003, NO1432 Ås, Norway

$9 \quad \underline{\text { liviu.ene@nmbu.no }}$

10 Erik Næsset

Department of Ecology and Natural Resource Management, Norwegian University of Life Sciences,

12 P.O. Box 5003, N01432 Ås, Norway

13 erik.naesset@nmbu.no

15 Corresponding author: Victor Felix Strîmbu, Department of Ecology and Natural Resource

16 Management, Norwegian University of Life Sciences, P.O. Box 5003, N01432 Ås, Norway

Tel.+4797044678, victor.strimbu@nmbu.no 


\section{Abstract}

22 This study proposes a method to perform spatially consistent imputations of forest data to serve

23 simulation studies where spatial autocorrelation is expected to have an effect (e.g. sampling

24 simulations and forest scenario analysis). Starting with a nearest neighbour imputation, an

25 optimization process brings the spatially comprehensive data to a desired state, controlled by a

26 target semivariogram and a target histogram. The target values for both parameters may be

27 approximated using empirical data, and are combined in the objective function used by the

28 optimization algorithm. Here we demonstrate a case study using wall-to-wall airborne laser scanner

29 (ALS) data, satellite data and field observations for an 852 ha forest area in southern Norway.

30 Different combinations of data types and target parameters were trialled, and the target values

31 were reached in most cases. In some cases, with a more restrictive objective function, the

32 semivariogram did not completely converge to its target values, yet still had a convergence of at

33 least $93 \%$, expressed by the difference reduction between initial and target values. The results

34 recommend the proposed method as practical means to generate spatially explicit forest data when

35 particular distribution and well defined spatial dependence are required.

37 Key words: spatial imputations, autocorrelation, semivariogram, simulation 
38

39

40

41

42

\section{Introduction}

Forest ecosystems provide valuable services essential to our wellbeing. Whether it is wood production, climate change mitigation through carbon sequestration, or providing wildlife habitat and recreational services, timely and accurate information regarding the state and changes of forests is in increasing demand.

Nowadays, forest surveys based on a combination of remotely sensed and field data are common practice. Often, resource assessments based on such data serve as the initial state in forest planning and scenario analysis. Remote sensing technologies provide georeferenced, wall-to-wall auxiliary information that enhance the traditional field sampling, having the potential to reduce the cost (Gobakken et al. 2013) and increase the accuracy when estimating forest parameters of treatment units in operational forest management (Næsset 2004). Sometimes, assessing the gain of using auxiliary information or simply constructing a confidence interval around a resource estimate may be too complex to derive analytically. Similarly, assessing the impact of a certain forest management plan in monetary and other terms becomes nontrivial under interacting phenomena (Gustafson et al. 2000). Under these circumstances, a simulation approach is preferred.

Spatially explicit simulations have proved useful in the context of sampling, as well as in forest planning and forest scenario analysis. Acknowledging the unfeasibility to obtain a complete ground truth for large areas, artificial spatial populations with known parameters may serve as surrogates for simulation studies. The artificial population serves as reference for statistical analyses in the context of sampling and estimation, or as the initial state in forest scenario analysis. The present work is motivated by the importance of spatially consistent populations for simulations where autocorrelation and spatial patterns are expected to have an effect.

Simulations performed prior to sampling, typically aim to optimize the field sampling effort by balancing information with cost. For example, Grafström et al. (2014) used simulations to show that a more representative field sample can be selected if the field plots are well spread in the auxiliary 
63 space (i.e. the range of values coming from remotely sensed data). Simulations can also tackle the 64 problem of forest inventory optimization (Tokola and Shrestha 1999), a notable example being the 65 establishment of the first national forest inventory in Tanzania (Tomppo et al. 2014). For instance, in clustered sampling, deciding upon the number of plots per cluster as well as the distance between them is a trade-off between new information and measurement cost. Adding an extra plot to the cluster is inexpensive but the information gain may be minor, whereas an additional cluster is expensive to measure, but with substantial information gain. Supporting this kind of decisions with simulations requires an artificial population with realistic spatial behaviour where information propagates spatially.

Post-sampling simulations have been used to assess the statistical properties of certain estimators when the complexity of the sampling design renders analytical evaluation impossible. In complex systematic sampling designs, which is often the case in forest surveys, approximating the estimator accuracy must resort to using conservative variance estimators that assume an underlying simple random sampling design. Simulation studies have shown that these estimators overestimate the variance, being unable to account for the so-called design effect (Ene et al. 2012; Ene et al. 2013; Saarela et al. 2015). The spatial autocorrelation is expected to further amplify the design effect, and was not incorporated in the previous simulation studies.

Simulative approaches are also often used in forest planning and forest scenario analysis (Gustafson et al. 2000). Here, we see an increasing interest toward greater spatial detail of forest representation (i.e. grid cell instead of forest stand) in the projection phase (Lämås and Eriksson 2003) as well as in the decision phase (Packalén et al. 2011). Spatial consistency can be a key aspect when forest scenario analysis integrates spatially interactive variables such as natural or human induced disturbance (James et al. 2011) or biodiversity (Marzluff et al. 2002), and especially when the treatment units in forest planning are raster cells, rather than entire forest stands (Packalén et

87 al. 2011). 
Spatial forest data can be generated through imputations using inexpensive remote sensing data and field plot data providing the forest variables of interest in addition to those derived from remotely sensed data. The terms carrier data and reference data are often used for the two data categories (Holmström et al. 2001). The missing values for the variables of interest in the carrier data (remotely sensed data) are taken from the reference set (field data) based on similarity between carrier variables values. While within-unit (e.g. stand or pixel) consistency between variables is preserved by certain imputation methods, spatial consistency between proximal units is usually compromised, meaning that the natural spatial relationship is lost. Efforts have been made to devise imputation methods that preserve the spatial consistency of the target population (Barth et al. 2012; Barth et al. 2009). Barth et al. (2009) describes a method to generate a spatially consistent population by optimizing an initial k-NN imputation. In this method, henceforth denoted as benchmark, the optimization is performed using simulated annealing (SA) with an objective function that quantifies relationships between adjacent pixels.

In the present paper, we take the aforementioned study further with the objective to generalize the notion of spatial consistency as well as to control the artificial population's composition. Here we use a target semivariogram to account for spatial neighbourhoods of arbitrary distance rather than just adjacent units, and a target histogram representing the frequencies of the imputed variable, to ensure a desired distribution. The second important objective was to validate the method in a real scenario, using remotely sensed data and field measurements as well as to find a way to set the target values for the selected criteria. The choice of this nonparametric approach (optimized imputations) over the more traditional geostatistical modelling (i.e. kriging (Liang 2012)) is motivated by the tendency of spatial predictions to produce surfaces that are too smooth, an incurable effect inherent to prediction that are based on weighted averages of spatially proximal observations. 
114

115

116

117

118

119

\section{Methods}

\subsection{Overview}

Analogous to the benchmark method (Barth et al., 2009), the algorithm starts with an initial imputation and then undergoes a process of spatial optimization. The initial state consists in a nearest neighbour (NN) imputation, which is iteratively optimized using SA, an approximation algorithm widely used when exhaustive search for an optimum solution is not feasible (Borges et al. 2014; Lockwood and Moore 1993). The SA objective function incorporates parameters that quantify spatial relationships as well as the composition of the population.

Once the population parameters are selected, the objective function is constructed as in Eq. 1:

(1) $\quad O=\sum_{i=1}^{m} w_{i} \frac{\mid \text { parameter }_{i}-\text { target_parameter }_{i} \mid}{\text { target_parameter }_{i}}$,

where $w_{i}$ is an optional weight factor for the $i$-th parameter. In the following sections, the parameters used in this study are described. For each unit in the spatial population, a list of $\mathrm{k}$ nearest neighbours is maintained. At each iteration in the SA optimization, a random unit is selected and a new imputed value is considered by selecting randomly one of the k neighbours. If the new value improves the current solution, it is kept, otherwise it is kept with a probability $p=$ $\exp \left(\operatorname{temp}^{-1}\left(O_{\text {new }}-O_{\text {current }}\right)\right)$, where temp is the current temperature, a value decreasing with every iteration, according to the annealing schedule (see section 2.3.2). In Barth et al. (2009) two parameters for quantifying the spatial variability are described. The first one is the short-range variance $(S R V)$, calculated as the average variance within all possible $3 \times 3$ blocks of cells:
(2)
$S R V=\frac{\sum_{i=1}^{n} \frac{\sum_{j=1}^{\operatorname{size}\left(b_{i}\right)}\left(x_{j}-\bar{x}\right)^{2}}{\operatorname{size}\left(b_{i}\right)-1}}{n}$.

In Eq. $2, i$ indexes the blocks and $j$ indexes cells within blocks. size $\left(b_{i}\right)$ is the number of cells in the $i-$ th block and may be smaller than 9 when the block is centred on the area boundary. The second parameter (Eq. 3) is the average pair-wise linear correlation between adjacent cells (Corr). A 4-way 
137 adjacency is used, with two sets of pairs being constructed for horizontal, respectively vertical

138 adjacency. For each cell, only the neighbours below and to the right are considered to avoid

139 redundancy. The correlation is calculated separately for each type of adjacency with the Corr

140 parameter being their average:

141

(3) $\quad \operatorname{Corr}=\frac{1}{2}\left(\frac{\operatorname{Cov}\left(X, X_{b}\right)}{\sqrt{\operatorname{Var}(X) \operatorname{Var}\left(X_{b}\right)}}+\frac{\operatorname{Cov}\left(X, X_{r}\right)}{\sqrt{\operatorname{Var}(X) \operatorname{Var}\left(X_{r}\right)}}\right)$,

where $X, X_{b}$, and $X_{r}$ are the variables with pair-wise cell observations $\left(x, x_{b}\right)$ and $\left(x, x_{r}\right)$, such that $x_{b}$ and $x_{r}$ are the cells bellow and to the right of $x$, respectively.

To ensure that the population mean does not drift away in the optimization process, the benchmark method uses a target mean as the third component in the objective function. Finally, the objective function combines the three parameters as shown in Eq. 4:

(4) $\quad O=\frac{\mid S R V-\text { target_SRV } \mid}{\text { target_SRV }}+\frac{\mid \text { Corr-target_Corr } \mid}{\text { target_Corr }}+\frac{\mid \text { Mean-target_Mean } \mid}{\text { target_Mean }}$.

\subsection{Proposed parameters}

The parameters used by the benchmark method are incomplete descriptions of both spatial variation and value distribution of imputed variables. $S R V$ and Corr only capture the variation of adjacent units, whereas in reality the variation increases as a function of distance. Similarly, the mean incompletely describes the population distribution. At the core of the current study there are two new parameters: a semivariogram (SV) to capture the population's spatial variation and a histogram (Histo) to control its distribution. The aim is to generate spatial data with values distributed according to a predefined histogram and with a spatial structure specified by a target semivariogram. When used together, these criteria provide a comprehensive description of a population's spatial relationship and distribution. As both the distribution and the semivariogram are continuous functions, integrating these criteria in the objective function requires discretization. For the distribution parameter, this is already done in the histogram through binning. In the case of semivariogram criteria, the observed empirical semivariogram must be compared against the target 
semivariogram model. As the empirical semivariogram is discretized into lag distance intervals, corresponding values for the target semivariogram are obtained by evaluating the semivariogram function at the centre of each lag interval. This means that for both criteria, there will be a vector of parameters instead of a single parameter.

The empirical semivariogram is obtained by calculating the variance of the difference between all pairs of values at a distance within a specified interval. The consecutive intervals may be identified by a lag index: $\operatorname{lag}_{k}=((k-1) \cdot \Delta \mathrm{h}, k \cdot \Delta \mathrm{h})$, where $\operatorname{lag}_{k}$ is the $k$-th lag, and $\Delta \mathrm{h}$ is the interval length. For the $k$-th lag the semivariance is calculated by:

169

(5) $\quad S V(k)=\frac{1}{2\left|P_{k}\right|} \sum_{\left(x_{i}, x_{j}\right) \in P_{k}}\left(x_{i}-x_{j}\right)^{2}$,

where $P_{k}$ denotes the set of all pairs of observations $\left(x_{i}, x_{j}\right)$ such that $\operatorname{dist}(i, j) \in \operatorname{lag}_{k}$. Integrating the semivariogram criteria into the objective function requires that $\Delta \mathrm{h}$ as well as the number of lags $l$ are specified. The total distance accounted for by the semivariogram is $l \times \Delta \mathrm{h}$. The semivariogram component of the objective function is constructed by summing up the individual contributions of each lag:

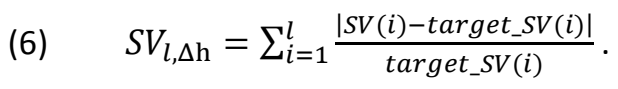

176 For the histogram, specifying the bin size is sufficient, as the number of bins $b$ is implicit when the 177 range of values is known. The histogram component of the objective function is:

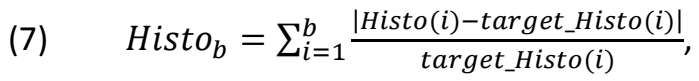
number of observations in the $i$-th bin. respective components. 


\subsection{Case study}

\subsubsection{Material}

The study site is a boreal forest in the municipality of Våler, Norway (Fig. 1). This 852 ha area is dominated by coniferous species, preponderantly Norway spruce (Picea abies (L.) Karst.) and Scots pine (Pinus sylvestris L.). Among the deciduous species, birch (Betula pubescens Ehrh.) has a more significant presence in the younger stands (Næsset et al. 2013a).

\section{Field data}

The field data used in this study comes from two different sample surveys carried out in the growing season of 2010. The first one is a systematic sample survey covering the entire area with 177 circular field plots laid out in the nodes of a grid with $150 \times 150 \mathrm{~m}$ spacing. There are several particularities to this systematic sampling design: 154 plots were located in young and mature forest stands and have a circular area of $400 \mathrm{~m}^{2}$, while the rest of 23 plots were located in recently regenerated stands. To reduce the amount of fieldwork, each of the 23 plots comprised four $20 \mathrm{~m}^{2}$ sub-plots placed $5.1 \mathrm{~m}$ away from the plot centre in each cardinal direction. The second survey dataset contained 923 sample plots located across 76 selected stands. These stands were subjectively chosen to represent different age classes, site productivity classes and species. Within each stand, between 5 and 16 plots were laid out systematically, their sizes varying according to forest conditions (see Table 1). Further details regarding the field protocol for these surveys can be found in (Næsset 2002; Næsset et al. 2013b). On each plot, the above ground biomass (AGB) of each measured tree was predicted using allometric equations (Marklund 1988) and then aggregated at the plot level and scaled to per hectare values (summary in Table 1). In this study, for the sake of simplicity, the plot-level errors associated with biomass estimation were ignored.

\section{Remotely sensed data}


Satellite imagery and airborne laser scanning (ALS) provided the remotely sensed data used in this

207 study. These spatially comprehensive data were the support for the artificial population, acting as

208 carrier in the imputations.

209 The satellite data consisted of a LANDSAT 7 scene acquired on 3 June, 2010. Using atmospherically

210 corrected NIR and Red bands, Normalized Difference Vegetation Index (NDVI) (Fig. 3 top) was

211 calculated. NDVI is a traditional vegetation index correlated with vegetation density, expected to

212 carry information about AGB.

The ALS data were acquired on 2 July, 2010 with an Optech ALTM Gemini laser scanner mounted on a Piper PA-31-310 Navajo aircraft. The mean ground pulse density was $7.3 \mathrm{~m}^{-2}$. The contractor, Blom Geomatics, Norway, processed the data using TerraScan software, and the ground points were classified using the progressive densification TIN algorithm. Height percentiles and canopy density metrics were calculated for each unit, and the metric most correlated with $A G B$, the $40^{\text {th }}$ height percentile (P40), was selected for further use in the study (Fig. 2).

A spatial resolution of $15 \times 15 \mathrm{~m}$ was used for the remotely sensed data to match the average field plot size. As LANDSAT 7 imagery has a pixel size of $30 \mathrm{~m}$, the original images were resampled to $15 \mathrm{~m}$ using bilinear interpolation. Similarly, the ALS variables were extracted on the same $15 \times 15 \mathrm{~m}$ grid. A forest stand map was used to mask out the non-forest pixels within the study area, resulting in 31385 population elements (forest pixels).

\subsubsection{Experiments}

Three types of imputation were performed (Table 2), each involving different variables. For each

type of imputation, the SA optimization was run 10 times (with different seeds) to verify the solution stability. The annealing schedule started with a temperature of 0.1 , had a cooling factor of 0.99 , and stopped at a temperature of $10^{-7}$. With 1000 iteration per temperature level, a total of 1375000 iterations were executed. The algorithm was implemented as a Java application allowing the user to select the input data, set the parameters, as well as visualize the solution. Attentive algorithm 
231 implementation resulted in fast processing time: 1375000 iterations in 2 minutes, on an Intel Core

232 i7-4910MQ @ $2.9 \mathrm{GHz}$. The main computation optimization was to update the parameter values at 233 each iteration instead of recalculating them. For instance, if the value of a single pixel changes, the 234 semivariogram is updated according to a finite number of data pairs that contain that particular 235 location.

236 First, the NDVI image was used as carrier and P40 as target variable (Table 2). Here P40 was used as 237 a proxy for AGB in the absence of a wall-to-wall biomass map. As P40 was available wall-to-wall, 238 target values for all parameters may be directly calculated. This enabled us to compare the results obtained using the benchmark parameters with those obtained using the new parameters.

To assess a more likely scenario, the second and third type of imputation had AGB as the target variable, with NDVI and P40 serving as carriers. The two different carriers were representative of situations with low and high correlation of the remotely sensed data with the forest attribute of interest. As wall-to-wall AGB was not available, the target values for the benchmark spatial consistency parameters were not known and they were therefore not tested here. The target semivariogram and histogram however, can be estimated by combining information from the field data with information from the carrier (see the next section).

Three different objective functions were tested. The first (Eq. 4) was the one proposed by Barth et al. (2009), which combines two spatial consistency criteria with the target mean (SRV + Corr + Mean). The second objective function (Eq. 8) sets targets for the semivariogram and the mean (SV + Mean), and lastly, the third objective function (Eq. 9) incorporates the semivariogram and the histogram (SV + Histo). When AGB was the variable of interest, weight factors of 2 (NDVI carrier) and 8 (P40 carrier) were used for the Mean component of the objective function ( $w_{\text {Mean }}$ in Eq. 8). The weight is needed here to balance the multi term SV criteria with the single term Mean. The values of $w_{\text {Mean }}$ were determined empirically after several trials.

255

(8) $\quad O=\sum_{i=1}^{l} \frac{\mid S V(i)-\text { target_SV }(i) \mid}{\text { target_SV }(i)}+w_{\text {Mean }} \frac{\mid \text { Mean-target_Mean } \mid}{\text { target_Mean }}$ 
(9) $\quad O=\sum_{i=1}^{l} \frac{\mid S V(i)-\text { target_SV }(i) \mid}{\text { target_SV }(i)}+\sum_{i=1}^{b} \frac{\mid H i s t o(i) \text {-target_Histo }(i) \mid}{\text { target_Histo }(i)}$

257 The results are evaluated according to the convergence percentage (Eq. 10), that measures how

258 close a particular parameter $\theta$ has come to its target value $\theta_{t}$ relative to its initial value $\theta_{i}$. In Eq. 10

259 the parameter's final value (i.e. following optimization) is denoted by $\theta_{f}$.

$260 \quad(10) \quad \%$ convergence $=\left(1-\frac{\left|\theta_{f}-\theta_{t}\right|}{\left|\theta_{i}-\theta_{t}\right|}\right) \times 100$

261 For all the experiments described above, the semivariogram was calculated using 20 lags of $30 \mathrm{~m}$

$262\left(S V_{20,30}\right)$, accounting for a total distance of $600 \mathrm{~m}$.

263 For the NDVI to AGB imputation we also tested target semivariograms with coarser approximations:

2643 lags of $200 \mathrm{~m}\left(S V_{3,200}\right), 6$ lags of $100 \mathrm{~m}\left(S V_{6,100}\right)$, and 12 lags of $50 \mathrm{~m}\left(S V_{12,50}\right)$, as well as target

265 semivariograms that account for shorter total distances: 5 lags of $30 \mathrm{~m}\left(S V_{5,30}\right), 10$ lags of $30 \mathrm{~m}$

$266\left(S V_{10,30}\right)$, and 15 lags of $30 \mathrm{~m}\left(S V_{15,30}\right)$. The resulting semivariograms were then compared against

267 the finer $S V_{20,30}$ target with the intention to determine the effect of the level of detail in the target

268 semivariogram. Consequently, the convergence is reported with respect to the $S V_{20,30}$ instead of

269 the actual target used for optimization.

270 In all experiments, the histogram had an interval width of $2 \mathrm{~m}$ for the $\mathrm{P} 40$ imputations, and $50 \mathrm{t} / \mathrm{ha}$

271 for the AGB imputations. As the right tail of the P40 wall-to-wall distribution ( 50 observations $>20 \mathrm{~m}$ )

272 was not represented in the reference set, the last two corresponding histogram bins were not

273 included in the Histo component. The histogram had 10 bins $\left(\right.$ Histo $\left._{10}\right)$ for both P40 and AGB

274 imputations.

\subsubsection{Setting the target parameters}

In Barth et al. (2009) the issue of setting the target values for the parameters was not addressed, as in a real scenario, the ground truth for the $Y$ variable is unknown. Here we show a way to set the target values for the AGB semivariogram and histogram parameters. 
279 The target semivariogram parameters were estimated by combining properties of the field sample 280 with properties of the full coverage auxiliary data. In this study, after exploratory analyses, we 281 decided to use the exponential semivariogram model:

(11) $\quad \gamma(h)=\tau^{2}+\sigma^{2}\left(1-e^{-\frac{|h|}{\varphi}}\right)$

The exponential semivariogram model has three parameters:

$\tau^{2}$, the so called nugget effect is the $y$-intercept and is sometimes interpreted as a combination of measurement error and microscale effect (Webster and Oliver 2004).

$\sigma^{2}$ is the partial sill. When added to the nugget effect, forms the sill $\left(\tau^{2}+\sigma^{2}\right)$, which can be seen as the scale parameter. It may also be interpreted as the variance of independent observations (i.e. separated by a distance greater that the autocorrelation range).

$\varphi$ is the range parameter and it relates to how far apart observations become independent. In the case of exponential semivariogram, the so called practical (or effective) range is $3 \varphi$, and denotes the distance at which the semivariogram reaches $95 \%$ of the sill.

According to Barnes (1991), the sample variance is a reasonable estimate of the semivariogram sill if the sample is evenly distributed over an area at least three times greater than the semivariogram range. Our study area spans approximately 1000 ha of land, thus large enough to cover three times the autocorrelation range of roughly $200 \mathrm{~m}$, typical to this type of forest (Næsset et al. 2013b).The variance of the 177 systematic plots $\left(S_{A G B_{-} S Y S}^{2}\right)$ was used to estimate the AGB sill $\left(\tau_{A G B}^{2}+\sigma_{A G B}^{2}\right)$. To get individual values for $\tau_{A G B}^{2}$ and $\sigma_{A G B}^{2}$, as well as an estimate for $\varphi_{A G B}$, we relied on the observation that P40 and AGB have a very similar spatial behaviour, as revealed by their empirical semivariograms calculated with the reference set of observations (Fig. 4). We assumed that the nugget-sill ratio and range parameter are similar for $\mathrm{AGB}$ and $\mathrm{P} 40: \tau_{A G B}^{2} / \sigma_{A G B}^{2} \approx \tau_{P 40}^{2} / \sigma_{P 40}^{2}$ and following parameters (values shown in Fig. 4): 
303

304

305

(12) $\quad \tau_{A G B}^{2}=\frac{S_{A G B S S Y S}^{2} \tau_{P 40}^{2}}{\tau_{P 40}^{2}+\sigma_{P 40}^{2}}$

(13) $\sigma_{A G B}^{2}=\frac{S_{A G B_{S} S Y S}^{2} \sigma_{P 40}^{2}}{\tau_{P 40}^{2}+\sigma_{P 40}^{2}}$

(14) $\varphi_{A G B}=\varphi_{P 40}$

The target histogram was calculated by scaling the value frequencies of the reference set $(n=1100)$ to the population size $(\mathrm{N}=31385)$. Here we chose to use the whole reference set to avoid having empty histogram bins. To be consistent with the histogram, the target mean was also calculated as the mean of all reference field plots.

\section{Results and discussion}

\subsection{Imputations of ALS data as biomass proxy}

In Table 3, the results are presented for each parameter in terms of mean and standard deviation across the 10 runs. Here, only values for $S V_{20,30}$ and $H i s t o_{10}$ are reported with corresponding targets as 0 . Detailed results at the level of individual lags for semivariogram and individual bins for histogram are seen in Fig. 5 and Fig. 6 respectively. The results may be visually compared in Fig. 7 (first column).

In general, the parameters reached the target values when they were included in the objective, and the solution was stable (i.e. small variation across the 10 runs). When the objective included the histogram, the semivariogram objective was mildly affected, preponderantly in the first lag (Fig. 5). Being closely connected to the first lag semivariance, $S R V$ and $C o r r$, although considerably ameliorated, did not reach their targets. The Mean criterion was satisfied regardless of how the objective function was constructed.

As expected, setting targets for $S R V$ and $C o r r$ ensured that the semivariance in the first lag also met the target (Fig. 5). In fact, semivariance was quite close to the target within the first $150 \mathrm{~m}$ $\left(S V_{5,30}=0.074\right)$, after which it started accumulating difference up to $S V_{20,30}=0.501$ for all 20 lags. 
326 This corresponds to a reduction of $78.6 \%$ in $S V_{20,30}$ (from the initial NN imputation), compared to

$32793.8 \%$ and $99.9 \%$ when the semivariogram was included in the objective, with and without the

328 histogram criteria, respectively.

329 Spatial optimization seemed to improve the pixel level prediction as well. The prediction mean

330 absolute error (MAE), calculated as the average absolute difference between the imputed value and

331 the true value, dropped from $4 \mathrm{~m}$ for the NN imputation to less than $3.7 \mathrm{~m}$ for the spatially

332 optimized imputations. This suggests that the spatial neighbourhood of a unit has explanatory power

333 that supplements the carrier information. Despite being a minor improvement, this result is

334 important as it shows that this spatial optimization method does not affect the local structure of the 335 population.

\subsection{Biomass imputations}

The results for the NDVI to AGB imputations are summarized in Table 4, and those for P40 to AGB in

Table 5. A visual comparison is provided by Fig. 7 (second and third columns). The $S R V$ and Corr

spatial consistency parameters are also reported, albeit their true value not being known.

When the objective was formulated by SV+Mean, both parameters reached their target regardless of the carrier used in the imputations.

Under the SV+Histo objective, the target histogram was successfully preserved for both NDVI (Fig. 9, top) and P40 (Fig. 9, bottom) carriers, but impeded the semivariance from reaching its target in the first lag with the NDVI carrier (Fig. 8, top). Here, the first lag accounted for $92 \%$ of the total difference (cumulated over all lags), and the first four lags $(120 \mathrm{~m}$ ) for $99 \%$. The semivariance in the first lag was $14 \%$ larger than the target. For the rest of the lags, this figure was below $1 \%$. With the P40 carrier, the semivariogram target was also met (Table 5, Fig. 8, bottom).

The number of lags as well as the lag distance are important when setting the target semivariogram. 
351 rate, and large lag intervals did not capture the short-range spatial dependence. The semivariogram

352 is a monotonically increasing function, causing the average semivariation over a distance interval to

353 be located around the middle of the interval. For this reason, when the objective function did not

354 include a target histogram (Fig. 10, top-left), the semivariogram had a periodic behaviour, matching

355 the finer scale target for distances marking the middle of the coarse lag intervals. This was most

356 evident with the coarse $200 \mathrm{~m}$ lags target semivariogram, where the resulting semivariogram

357 intersected the finer $S V_{20,30}$ target near the 100, 300, and $500 \mathrm{~m}$ lag distances. Including a target

358 histogram (Fig. 10, top-right), removed this effect, and alleviated the differences for far distance lags

359 at the expense of further degrading the semivariance within smaller distances (i.e. $<100 \mathrm{~m}$ ).

360 As anticipated, setting a target semivariogram that covers a limited distance (Fig. 10, bottom row)

361 secured the convergence of semivariance for the close range lags. Still, the semivariance beyond the

362 range covered by the target was close to the desired values for $S V_{10,30}$ and $S V_{15,30}$. In the case of

363 the $S V_{5,30}$ target however, the semivariance for distances greater that $150 \mathrm{~m}$ was considerably

364 below the values of the $S V_{20,30}$ target, with an overall convergence of only $46 \%$ (Fig. 10 bottom-

365 left). This figure increased to $89 \%$ when the target histogram was combined with $S V_{5,30}$ in the

366 objective (Fig. 10, bottom-right). In this case, the histogram compensated the lack of target values

367 for the far distances in the $S V_{5,30}$ target. The histogram constrained the imputed values to follow a

368 certain distribution, effectively setting an implicit target on the semivariogram sill. This result is

369 interesting as it shows the interdependence between the semivariogram sill and the distribution of

370 spatial data. When combined with the $S V_{10,30}$ and $S V_{15,30}$ targets, the histogram did not improve

371 the semivariogram convergence. On the contrary, the semivariogram convergence dropped from

$37292 \%\left(S V_{10,30}+\right.$ Mean $)$ and $98 \%\left(S V_{15,30}+\right.$ Mean $)$ to $89 \%\left(S V_{10,30}+\right.$ Histo $\left._{10}\right)$ and $93 \%\left(S V_{15,30}+\right.$ Histo $\left._{10}\right)$.

373 Here, the target semivariograms were already specified up to a distance close to the sill and the

374 additional histogram parameters only introduced additional constraints. Regardless of the effect on

375 the semivariogram convergence, the histograms themselves always reached their respective targets. 
In summary, using too large lag distances will deteriorate the semivariogram for short distances, and using too few lags will deteriorate the semivariogram for the far distances. While the missing target values for the semivariation near the sill in the latter case may be compensated by setting a target histogram, the result of using a coarse target semivariogram will not be improved by the histogram. In general, the short-range semivariation target is reached with more difficulty and is prone to give in to additional constrains. Depending on the application, this may have unwanted effects and the user should consider relaxing some of the constrains. One way to improve the method based on the above findings is to use unequal lag intervals to approximate the target semivariogram. For instance, the semivariogram could be binned based on equal semivariation intervals ( $Y$-axis) instead of equal distance intervals (X-axis). This may improve the semivariogram approximation, and in the same time reduce the number of target parameters with the potential to improve solution convergence in the optimization process. Another improvement worth investigating is expanding the set of reference data by fitting a copula function to the initial set (Ene et al. 2012). In this manner, more values are available in the optimization process, making it easier to reach a desired configuration.

\section{Conclusion}

Barth et al. (2009) proposed a method to obtain spatially consistent forest data through imputations and spatial optimization. In the current study we have taken this approach further by incorporating a target semivariogram and histogram into the simulated annealing objective function, with the aim to control the spatial structure as well as the distribution of the imputed values. Furthermore, we devised a practical way to set the values of these parameters and to test the method in a real scenario, using remotely sensed and field data. Despite the complexity of the objective function, the solution converged to the prescribed structure in relatively short time. Thus, the proposed methodology offers a practically feasible means to create spatially explicit artificial populations to be used in management scenario analyses as well as sampling simulations over large forest landscapes. 
402

403

404

405

406

407

408

409

410

411

412

413

414

415

416

417

418

419

420

421

422

423

424

425

426

427

428

429

430

431

432

433

434

435

436

437

438

439

440

441

442

443

444

445

446

447

448

449

Barnes, R.J. 1991. The variogram sill and the sample variance. Mathematical geology 23(4): 673-678. doi: 10.1007/BF02065813.

Barth, A., Lind, T., and Ståhl, G. 2012. Restricted imputation for improving spatial consistency in landscape level data for forest scenario analysis. Forest Ecology and Management 272(0): 61-68. doi: http://dx.doi.org/10.1016/j.foreco.2011.07.009.

Barth, A., Wallerman, J., and Stahl, G. 2009. Spatially consistent nearest neighbor imputation of forest stand data. Remote Sensing of Environment 113(3): 546-553. doi: http://dx.doi.org/10.1016/j.rse.2008.09.011.

Borges, P., Bergseng, E., and Eid, T. 2014. Adjacency constraints in forestry - a simulated annealing approach comparing different candidate solution generators. Mathematical \& Computational Forestry \& Natural Resource Sciences 6(1): 11-25.

Ene, L.T., Næsset, E., Gobakken, T., Gregoire, T.G., Ståhl, G., and Holm, S. 2013. A simulation approach for accuracy assessment of two-phase post-stratified estimation in large-area LiDAR biomass surveys. Remote Sensing of Environment 133(0): 210-224. doi:

http://dx.doi.org/10.1016/j.rse.2013.02.002.

Ene, L.T., Næsset, E., Gobakken, T., Gregoire, T.G., Ståhl, G., and Nelson, R. 2012. Assessing the accuracy of regional LiDAR-based biomass estimation using a simulation approach. Remote Sensing of Environment 123(0): 579-592. doi: http://dx.doi.org/10.1016/j.rse.2012.04.017.

Gobakken, T., Korhonen, L., and Næsset, E. 2013. Laser-assisted selection of field plots for an areabased forest inventory. Silva Fennica 47(5). doi: doi:10.14214/sf.943.

Grafström, A., Saarela, S., and Ene, L.T. 2014. Efficient sampling strategies for forest inventories by spreading the sample in auxiliary space. Canadian Journal of Forest Research 44(10): 1156-1164. doi: 10.1139/cjfr-2014-0202.

Gustafson, E.J., Shifley, S.R., Mladenoff, D.J., Nimerfro, K.K., and He, H.S. 2000. Spatial simulation of forest succession and timber harvesting using LANDIS. Canadian Journal of Forest Research 30(1): 32-43.

Holmström, H., Nilsson, M., and Ståhl, G. 2001. Simultaneous Estimations of Forest Parameters using Aerial Photograph Interpreted Data and the $k$ Nearest Neighbour Method. Scandinavian Journal of Forest Research 16(1): 67-78. doi: 10.1080/028275801300004424.

James, P.M.A., Fortin, M.J., Sturtevant, B.R., Fall, A., and Kneeshaw, D. 2011. Modelling Spatial Interactions Among Fire, Spruce Budworm, and Logging in the Boreal Forest. Ecosystems 14(1): 60-75.

Lämås, T., and Eriksson, L.O. 2003. Analysis and planning systems for multiresource, sustainable forestry: The Heureka research programme at SLU. Canadian Journal of Forest Research 33(3): 500-508.

Liang, J. 2012. Mapping large-scale forest dynamics: a geospatial approach. Landscape Ecology 27(8): 1091-1108. doi: 10.1007/s10980-012-9767-7.

Lockwood, C., and Moore, T. 1993. Harvest scheduling with spatial constraints: a simulated annealing approach. Canadian Journal of Forest Research 23(3): 468-478. doi: 10.1139/x93-065.

Marklund, L.G. 1988. Biomass functions for pine, spruce and birch in Sweden. Swedish University of Agricultural Sciences, Department of Forest Survey, Umeå.

Marzluff, J.M., Millspaugh, J.J., Ceder, K.R., Oliver, C.D., Withey, J., McCarter, J.B., Mason, C.L., and Comnick, J. 2002. Modeling Changes in Wildlife Habitat and Timber Revenues in Response to Forest Management. Forest Science 48(2): 191-202.

Næsset, E. 2002. Predicting forest stand characteristics with airborne scanning laser using a practical two-stage procedure and field data. Remote Sensing of Environment 80(1): 88 - 99.

Næsset, E. 2004. Accuracy of forest inventory using airborne laser scanning: evaluating the first nordic full-scale operational project. Scandinavian Journal of Forest Research 19(6): 554 - 557. 
450

451

452

453

454

455

456

457

458

459

460

461

462

463

464

465

466

467

468

469

470

471

Næsset, E., Bollandsås, O.M., Gobakken, T., Gregoire, T.G., and Ståhl, G. 2013a. Model-assisted estimation of change in forest biomass over an 11 year period in a sample survey supported by airborne LiDAR: A case study with post-stratification to provide "activity data". Remote Sensing of Environment 128: 299-314. doi: 10.1016/j.rse.2012.10.008.

Næsset, E., Gobakken, T., Bollandsås, O.M., Gregoire, T.G., Nelson, R., and Ståhl, G. 2013b. Comparison of precision of biomass estimates in regional field sample surveys and airborne LiDAR-assisted surveys in Hedmark County, Norway. Remote Sensing of Environment 130(0): 108 $-120$.

Packalén, P., Heinonen, T., Pukkala, T., Vauhkonen, J., and Maltamo, M. 2011. Dynamic Treatment Units in Eucalyptus Plantation. Forest Science 57(5): 416-426.

Saarela, S., Schnell, S., Grafström, A., Tuominen, S., Nordkvist, K., Hyyppä, J., Kangas, A., and Ståhl, G. 2015. Effects of sample size and model form on the accuracy of model-based estimators of growing stock volume. Canadian Journal of Forest Research 45(11): 1524-1534. doi: 10.1139/cjfr2015-0077.

Tokola, T., and Shrestha, S.M. 1999. Comparison of cluster-sampling techniques for forest inventory in southern Nepal. Forest Ecology and Management 116(1-3): 219-231. doi: http://dx.doi.org/10.1016/S0378-1127(98)00457-5.

Tomppo, E., Malimbwi, R., Katila, M., Mäkisara, K., Henttonen, H.M., Chamuya, N., Zahabu, E., and Otieno, J. 2014. A sampling design for a large area forest inventory: case Tanzania. Canadian Journal of Forest Research 44(8): 931-948. doi: 10.1139/cjfr-2013-0490.

Webster, R., and Oliver, M.A. 2004. Geostatistics for environmental scientists. John Wiley \& Sons, LTD. 
Table 1. Field plot measurements for above ground biomass.

\begin{tabular}{lllllll}
\hline Forest class & \multicolumn{3}{l}{ Systematic survey } & \multicolumn{4}{l}{ Stand survey } \\
\cline { 2 - 7 } & $\begin{array}{l}\text { Plot area } \\
\left(\mathrm{m}^{2}\right)\end{array}$ & $\begin{array}{l}\text { Number } \\
\text { of plots }\end{array}$ & $\begin{array}{l}\text { AGB mean, st. } \\
\text { dev }(\mathrm{Mg} / \mathrm{ha})\end{array}$ & $\begin{array}{l}\text { Plot area } \\
\left(\mathrm{m}^{2}\right)\end{array}$ & $\begin{array}{l}\text { Number } \\
\text { of plots }\end{array}$ & $\begin{array}{l}\text { AGB mean, st. } \\
\operatorname{dev}(\mathrm{Mg} / \mathrm{ha})\end{array}$ \\
\hline Recently regenerated & $4 \times 20$ & 23 & $5.03,12.17$ & $4 \times 20$ & 178 & $5.79,15.23$ \\
Young & 400 & 42 & $112.32,52.1$ & 125 & 244 & $108.75,56.17$ \\
Mature spruce dominated & 400 & 62 & $150.11,61$ & 250 & 437 & $152.17,81.25$ \\
Mature pine dominated forest & 400 & 50 & $162.49,84.37$ & 250 & 64 & $121.32,50.14$ \\
Total & & 177 & $125.79,80.86$ & & 923 & $110.21,84.5$ \\
\hline
\end{tabular}

Table 2. Summary of the experiments

\begin{tabular}{|c|c|c|c|}
\hline Carrier & Variable of interest & Imputation methods & Target values \\
\hline NDVI & $\mathrm{P} 40$ & $\begin{array}{l}\text { NN } \\
\text { O (SRV + Corr+ Mean) } \\
\text { O (SV + Mean) } \\
\text { O (SV + Histo) }\end{array}$ & $\begin{array}{l}\text { Known from ground } \\
\text { truth }\end{array}$ \\
\hline NDVI & $A G B$ & $\begin{array}{l}\text { NN } \\
\mathrm{O}(\mathrm{SV}+\text { Mean) } \\
\mathrm{O} \text { (SV + Histo) }\end{array}$ & $\begin{array}{l}\text { Estimated from field } \\
\text { data and auxiliary }\end{array}$ \\
\hline P40 & $A G B$ & $\begin{array}{l}\text { NN } \\
\mathrm{O}(\mathrm{SV}+\text { Mean) } \\
\mathrm{O} \text { (SV + Histo) }\end{array}$ & $\begin{array}{l}\text { Estimated from field } \\
\text { data and auxiliary }\end{array}$ \\
\hline
\end{tabular}

Note: NN, nearest neighbour imputation; $\mathrm{O}$, optimized imputation with target parameters in parenthesis

Table 3. Results summary for NDVI to P40 imputations.

\begin{tabular}{lllllll}
\hline Method & SV $_{20,30}$ & Histo $_{10}$ & Mean $(\mathrm{m})$ & $\mathrm{SRV}\left(\mathrm{m}^{2}\right)$ & Corr & Prediction MAE $(\mathrm{m})$ \\
\hline NN & $2.279(0 \%)$ & $4.037(0 \%)$ & $7.80(0 \%)$ & $12.56(0 \%)$ & $0.364(0 \%)$ & 4.010 \\
O (SRV+Corr+Mean) & $0.501(78 \%)$ & $2.310(42.8 \%)$ & $\mathbf{6 . 7 8 ( 1 0 0 \% )}$ & $\mathbf{7 . 5 4}(\mathbf{1 0 0 \% )}$ & $\mathbf{0 . 7 1 2 ( 1 0 0 \% )}$ & 3.626 \\
O (SV+Mean) & $\mathbf{0 . 0 0 1 ( 9 9 . 9 \% )}$ & $2.119(47.5 \%)$ & $\mathbf{6 . 7 8 ( 1 0 0 \% )}$ & $\mathbf{7 . 5 5 ( 9 9 . 8 \% )}$ & $0.637(78.4 \%)$ & 3.637 \\
O (SV+Histo) & $0.14(93.8 \%)$ & $\mathbf{0 ( 1 0 0 \% )}$ & $\mathbf{6 . 7 5 ( 9 7 \% )}$ & $8.28(85.3 \%)$ & $0.579(61.8 \%)$ & 3.687 \\
Target & $0(100 \%)$ & $0(100 \%)$ & $6.78(100 \%)$ & $7.54(100 \%)$ & $0.712(100 \%)$ & - \\
\hline
\end{tabular}

Note: Values given as averages over 10 different runs (convergence percentage in parentheses). The parameters that constitute the objective are shown in italics, and those that have reached the target are shown in bold.

Table 4. Results summary for NDVI to AGB imputations.

\begin{tabular}{llllll}
\hline Method & $\mathrm{SV}_{20,30}$ & Histo $_{10}$ & Mean (Mg/ha) & SRV $\left(\mathrm{Mg}^{2} / \mathrm{ha}^{2}\right)$ & Corr \\
\hline NN & $2.965(0 \%)$ & $2.009(0 \%)$ & $113.22(0 \%)$ & 4865 & 0.334 \\
O (SV+Mean) & $\mathbf{0 . 0 3 1 ( 9 8 . 9 \% )}$ & $1.3(35.3 \%)$ & $\mathbf{1 1 2 . 7 2 ( 1 0 0 \% )}$ & 2717 & 0.640 \\
O (SV+Histo) & $0.154(94.8 \%)$ & $\mathbf{0 . 0 0 5 ( 9 9 . 8 \% )}$ & $\mathbf{1 1 3 . 5 1 ( - 5 8 \% )}$ & 2937 & 0.609 \\
Target & $0(100 \%)$ & $0(100 \%)$ & $112.72(100 \%)$ & - & - \\
\hline
\end{tabular}

Note: Values given as averages over 10 different runs (convergence percentage in parentheses). The parameters that constitute the objective are shown in italics, and those that have reached the target are shown in bold. 
Table 5. Results summary for ALS to AGB imputations.

\begin{tabular}{|c|c|c|c|c|c|}
\hline Method & $\mathrm{SV}_{20,30}$ & Histo $_{10}$ & Mean (Mg/ha) & $\operatorname{SRV}\left(\mathrm{Mg}^{2} / \mathrm{ha}^{2}\right)$ & Corr \\
\hline NN & $1.69(0 \%)$ & $2.999(0 \%)$ & $99.48(0 \%)$ & 2976 & 0.623 \\
\hline $\mathrm{O}$ (SV+Mean) & $0.016(99.1 \%)$ & $0.884(70.5 \%)$ & $112.72(100 \%)$ & 2680 & 0.690 \\
\hline $\mathrm{O}$ (SV+Histo) & $0.023(98.6 \%)$ & 0.004 (99.8\%) & $110.58(83.8 \%)$ & 2679 & 0.694 \\
\hline Target & 0 (100\%) & 0 (100\%) & $112.72(100 \%)$ & - & - \\
\hline
\end{tabular}

Note: Values given as averages over 10 different runs (convergence percentage in parentheses). The parameters that constitute the objective are shown in italics, and those that have reached the target are shown in bold. 
472

473 474 475

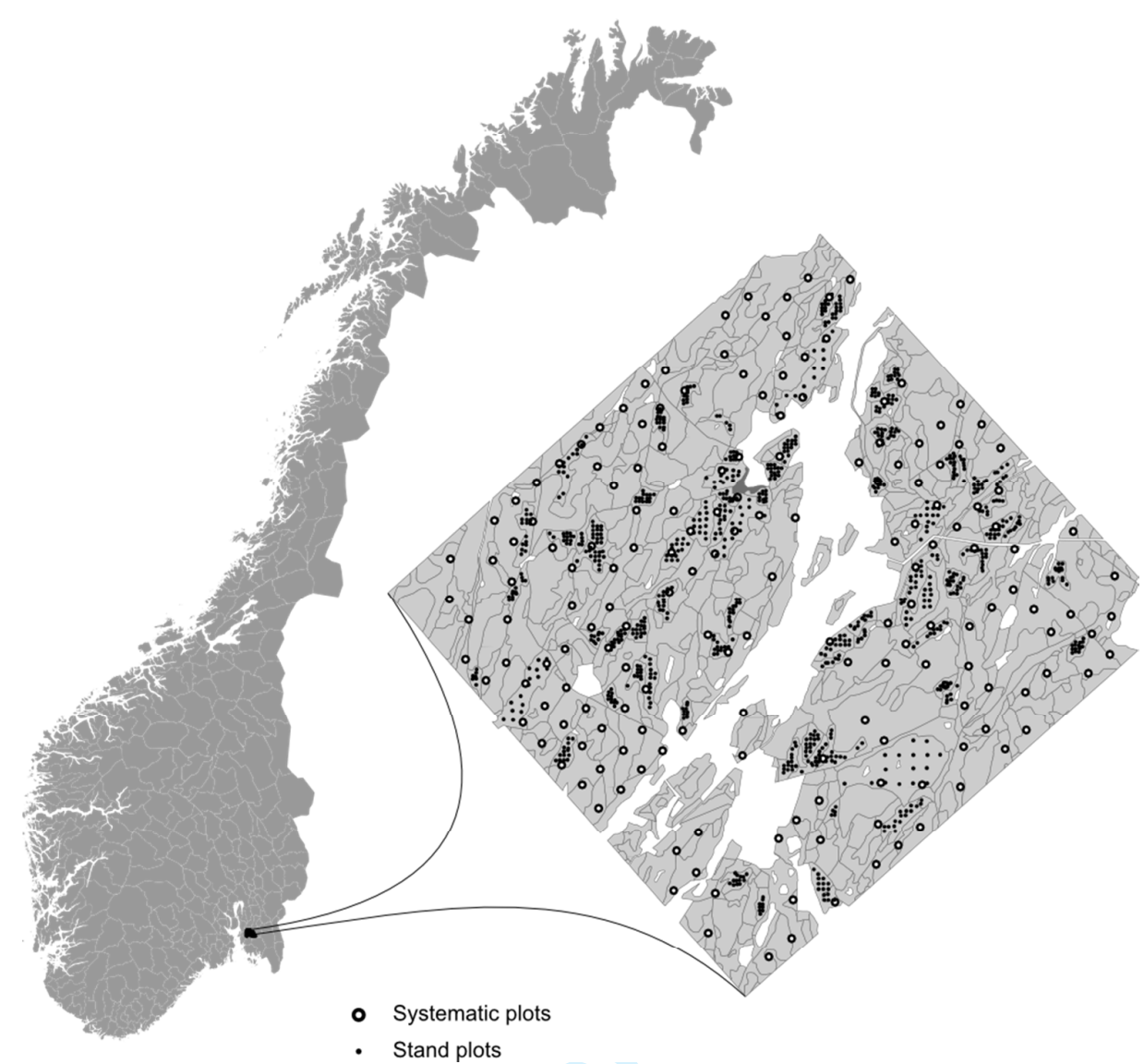

Figure 1.

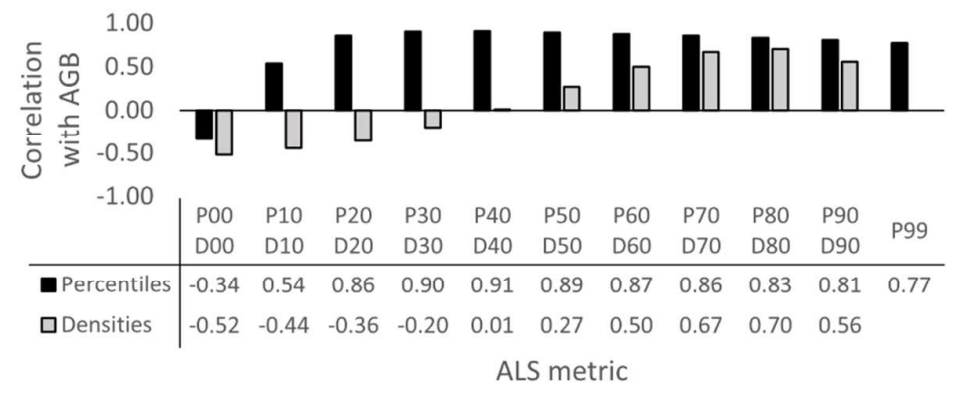

Figure 2. 

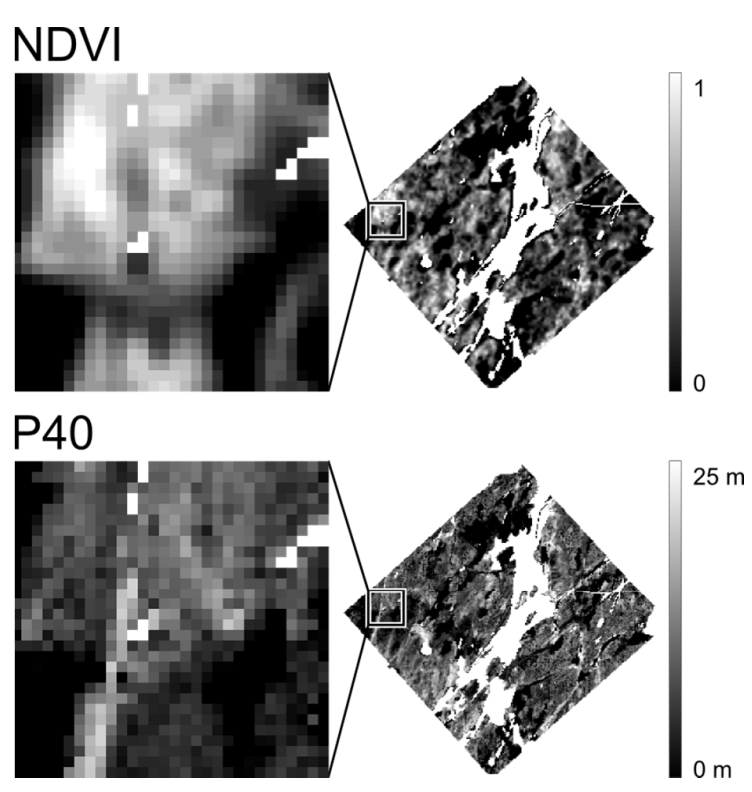

Figure 3.
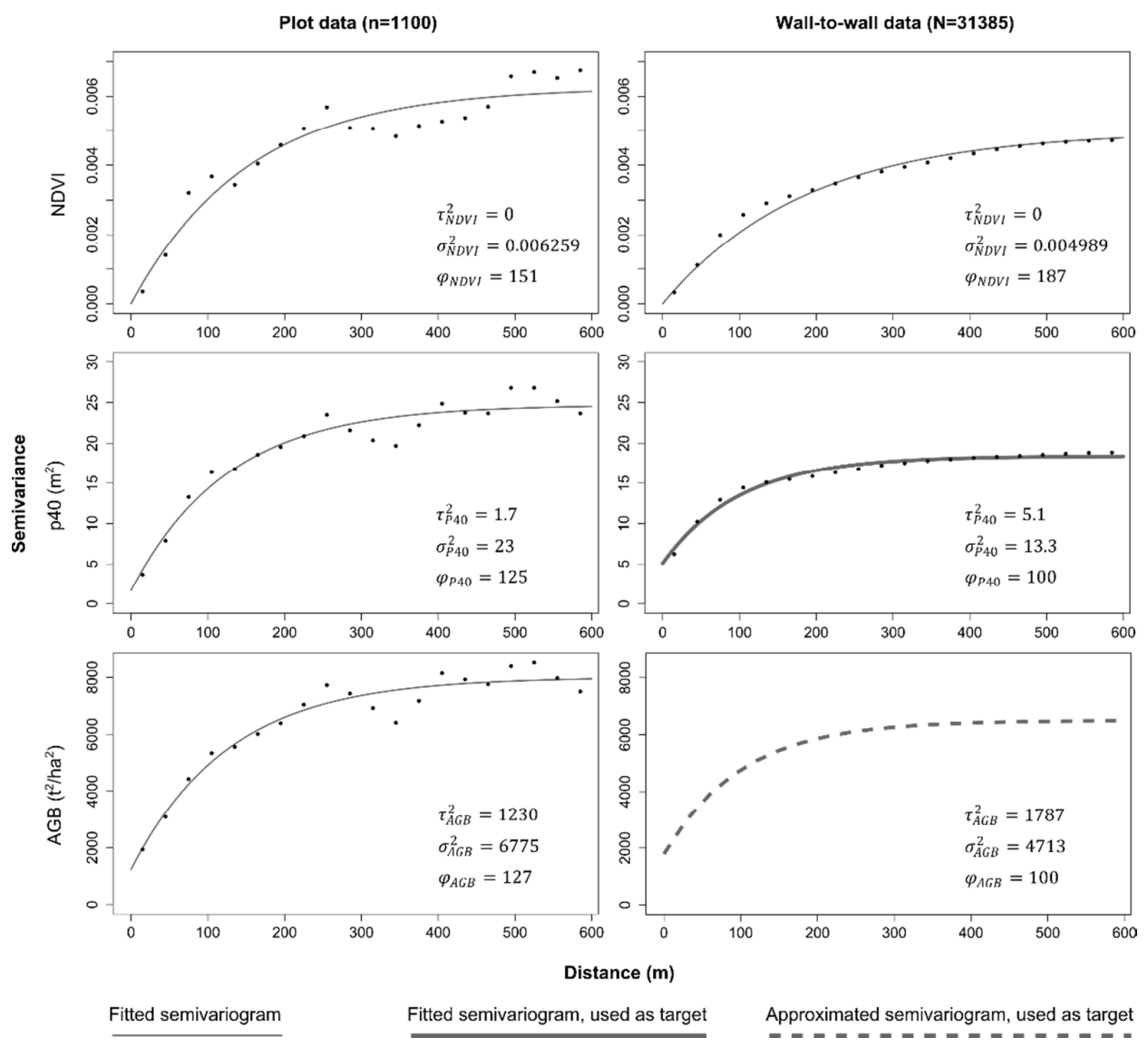

479

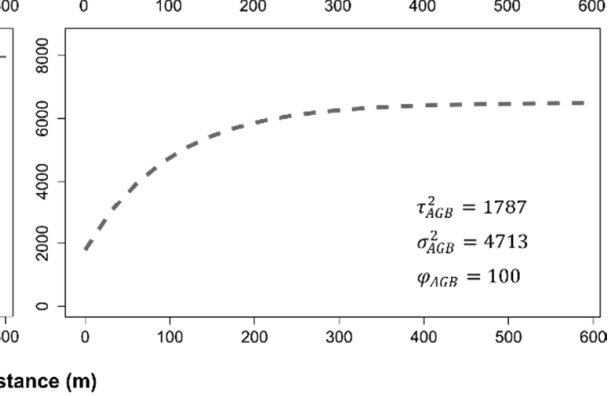

Figure 4. 




Figure 5.

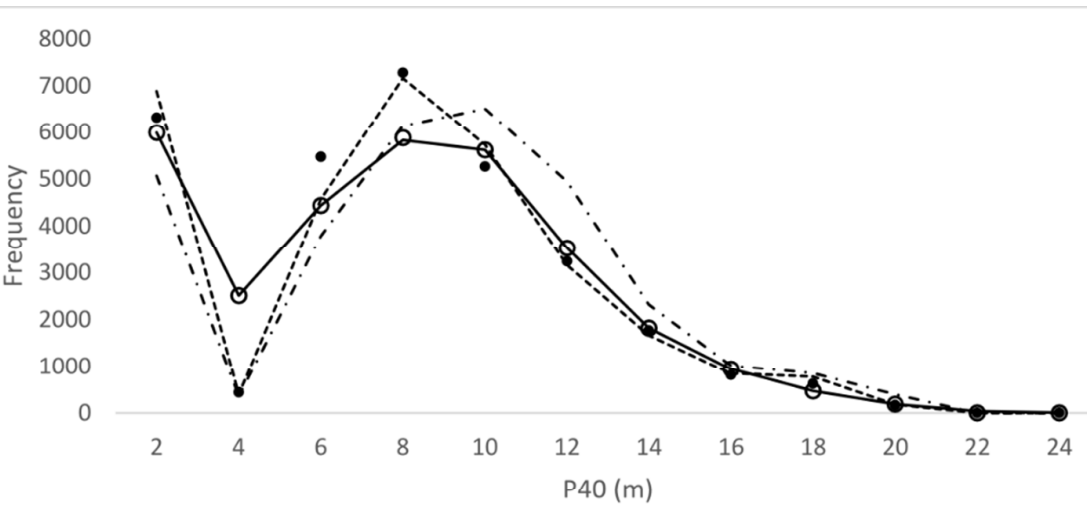

Figure 6. 

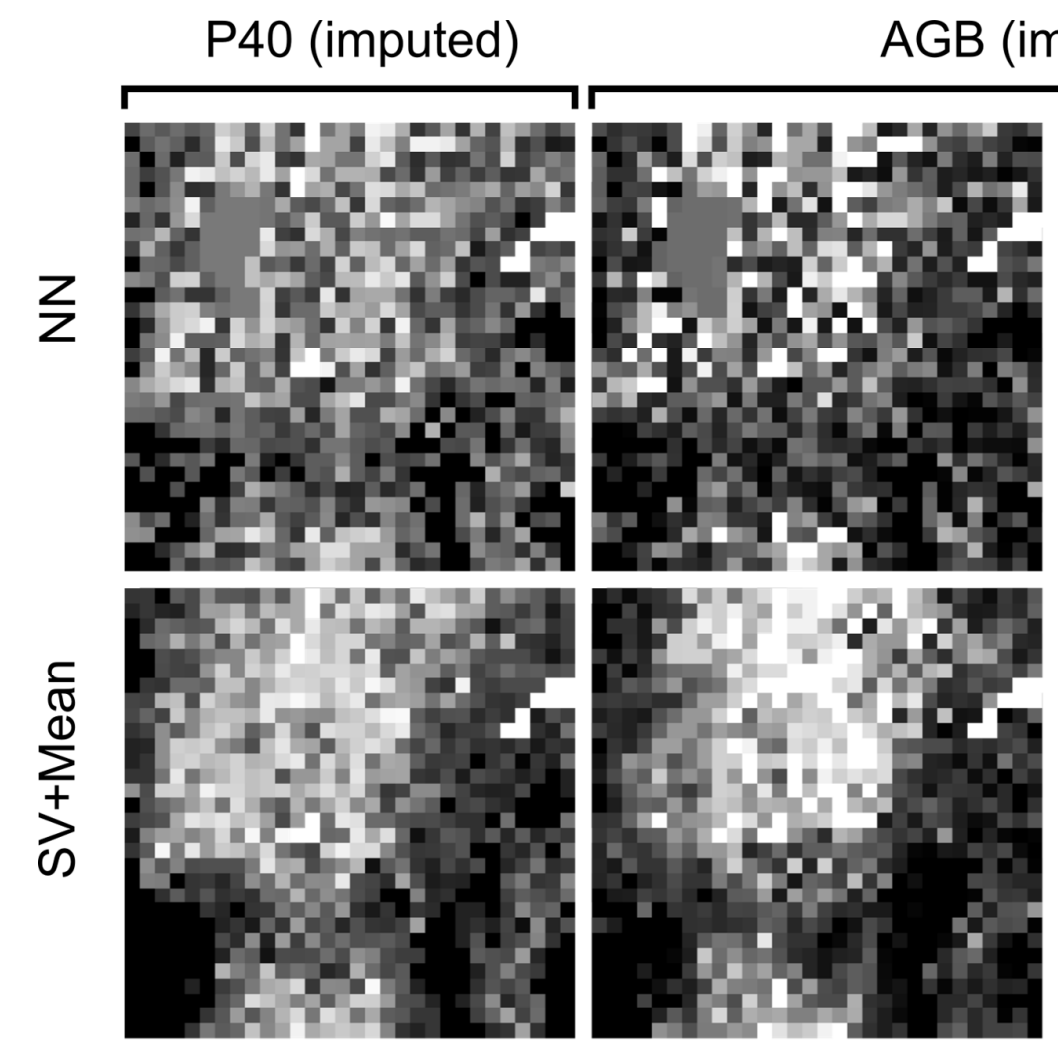

AGB (imputed)
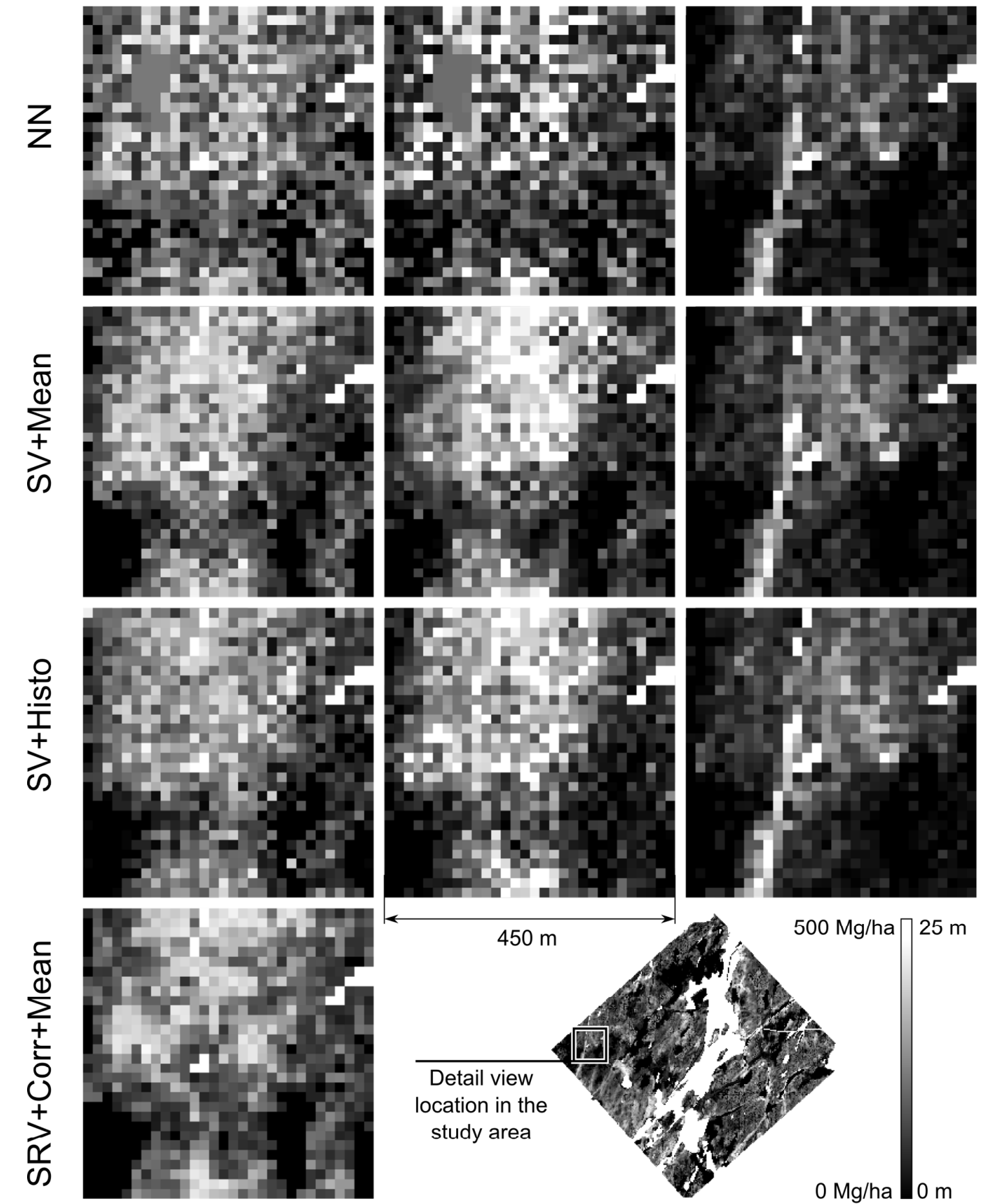

Figure 7. 



Figure 8. 


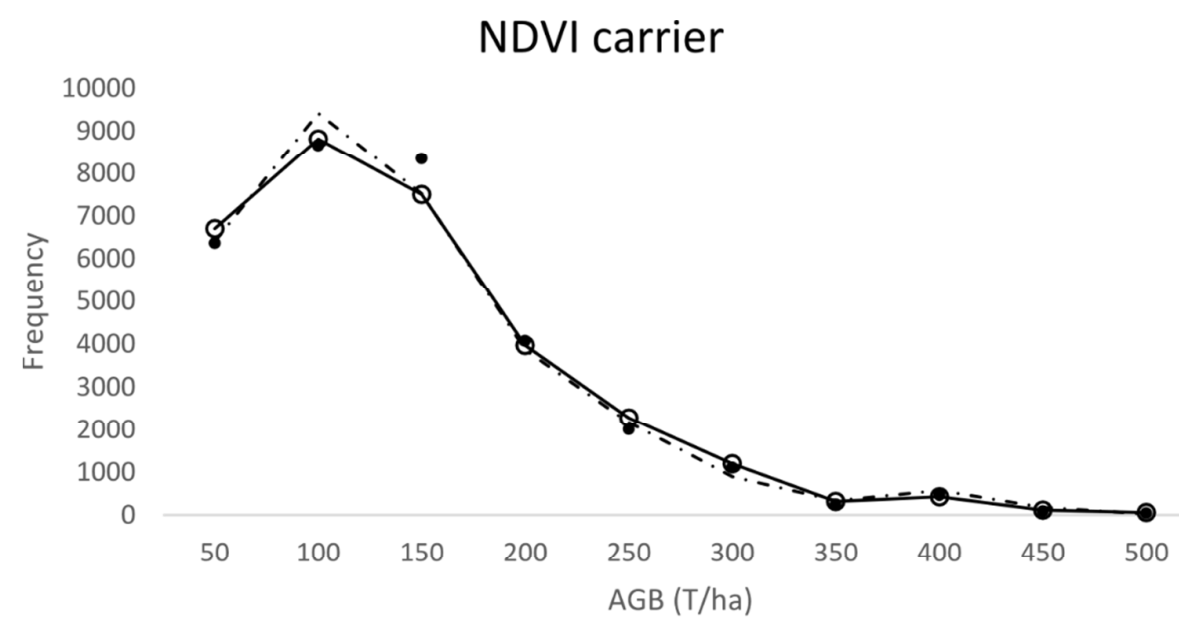

- Target $\quad-\cdot-\mathrm{NN} \quad \bullet \quad \mathrm{SV}+$ Mean $\quad \mathrm{O} \quad \mathrm{SV}+$ Histo

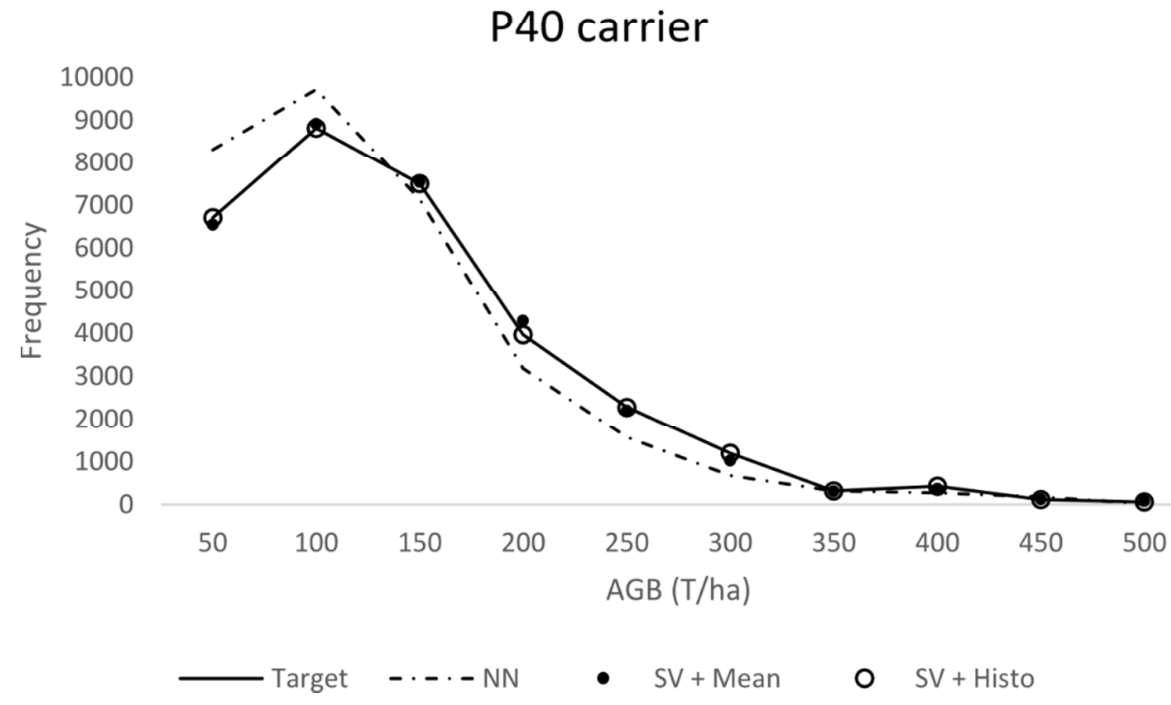

Figure 9. 
$S V+$ Mean

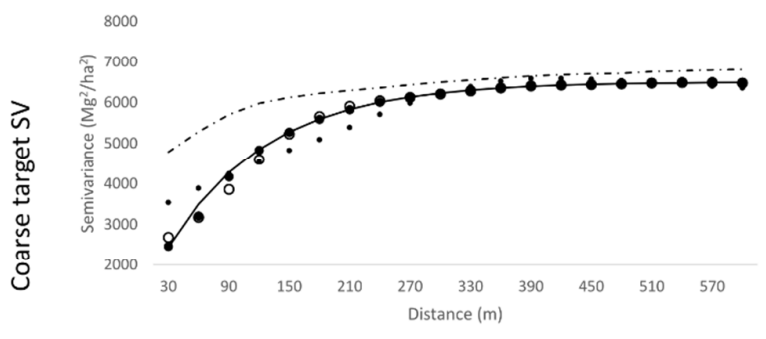

- ${ }_{3 \times 200} \mathbf{0}_{6 \times 100} \quad{ }_{12 \times 50}-20 \times 30$ Target $\quad \cdots \cdots \mathrm{NN}$

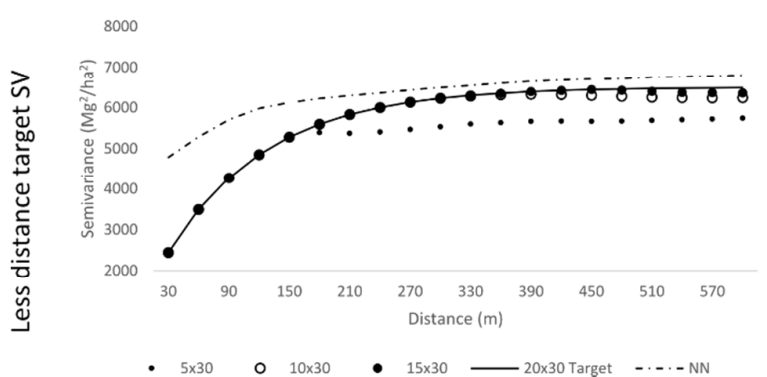

SV + Histo
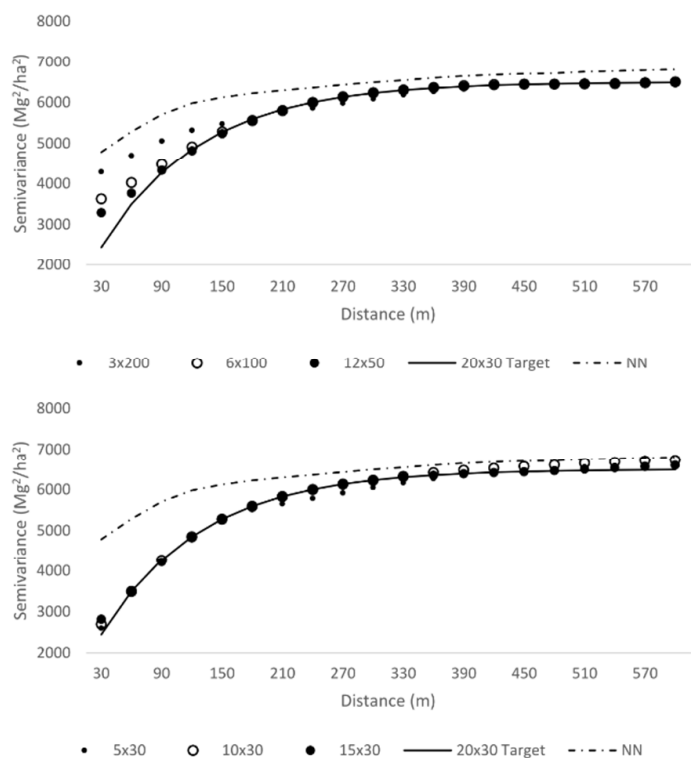

490

Figure 10. 
Fig. 1. Study area location and map of mainland Norway. The black dots represent the locations of the field plots for stand-wise inventory in selected stands, and the circles the locations of the field plots acquired in a systematic design over the entire study area. Grey lines are stand boundaries. Grey areas are forested, white is non-forest.

Fig. 2. Pearson's correlation coefficient between the ALS metrics and AGB, calculated at the field plot level

Fig. 3. The carriers used in this study: LANDSAT 7 NDVI and ALS $40^{\text {th }}$ height percentile (P40).

Fig. 4. Semivariograms for NDVI, P40, and AGB. The left column shows the semivariograms estimated with just the plot data, and the right column shows the semivariograms as calculated from wall-to-wall data (NDVI and P40) or estimated (AGB). The dots are the empirical semivariances for discrete lags of $30 \mathrm{~m}$ and the lines represent theoretical semivariograms fitted by the exponential model with parameter values given on the bottom right of each plot. The dashed line in the right, bottom panel is the semivariogram with approximated parameters, used as target for AGB imputations.

Fig. 5. Semivariograms of the imputed P40 variable for different imputation methods. The target semivariogram is shown with a solid line. NN is the initial nearest neighbour imputation; SRV+Corr+Mean, SV+Mean, and SV+Histo are spatially optimized imputations.

Fig. 6. Histograms of the imputed P40 variable for different imputation methods. The target histogram is shown with a solid line. NN is the initial nearest neighbour imputation; SRV+Corr+Mean, SV+Mean, and SV+Histo are spatially optimized imputations.

Fig. 7. Imputation results seen in a $450 \times 450 \mathrm{~m}$ window within the area. The P40 imputations are shown in the first column, and the AGB imputations in the second (NDVI carrier) and third (P40 carrier) columns. The rows separate imputation methods: the initial NN imputations are on the first row and spatially optimised imputations on the remaining rows. 
Fig. 8. Semivariograms of imputed AGB using NDVI (top), and P40 (bottom) carriers. The target semivariogram is shown with a solid line. NN is the initial nearest neighbour imputation; SV+Mean, and SV+Histo are spatially optimized imputations.

Fig. 9. Histograms of imputed AGB using NDVI (top), and P40 (bottom) carriers. The target histogram is shown with a solid line. NN is the initial nearest neighbour imputation; SV+Mean, and SV+Histo are spatially optimized imputations.

Fig. 10. Semivariograms for imputation results using different discretizations of the target semivariogram. Coarse target semivariograms: $\mathrm{SV}_{3,200}, \mathrm{SV}_{6,100}$, and $\mathrm{SV}_{12,50}$ (first row). Short ranged target semivariograms: $\mathrm{SV}_{5,30}, \mathrm{SV}_{10,30}$, and $\mathrm{SV}_{15,30}$ (second row). Additional targets are set for mean (first column), and histogram (second column). The finer $\mathrm{SV}_{20,30}$ target used throughout this study is shown with a solid line. 